\title{
Les lipides complexes du lait Aspects biologiques. Aspects technologiques
}

\author{
(MISE AU POINT)
}

\author{
par \\ S. KUZDZAL-SAVOIE et J. MOAL \\ Station Centrale de Recherches Laitières \\ et de Technologie des Produits Animaux, I.N.R.A., Jouy-en-Josas
}

\section{INTRODUCTION}

Le lait est une émulsion de matière grasse. La phase aqueuse est une solution de lactose et de sels contenant en suspension des matières protéiques.

Le lait de vache, seul lait concerné dans l'étude présente, contient en moyenne $35 \mathrm{~g}$ de matière grasse par litre.

Obtenue par des moyens mécaniques (écrémage du lait, barattage de la crème, fusion et centrifugation du beurre) cette matière grasse est constituée par 99 à 99,5 p. 100 de glycérides (triglycérides : 96-97 p. 100, diglycérides : 2-3 p. 100, monoglycérides 0,1 p. 100 environ) et 0,5 à 1 p. 100 de composés divers : lipides non glycéridiques (acides gras libres, esters de stérols, esters d'alcools aliphatiques à longues chaînes (cérides), traces de phospholipides) et substances non lipidiques liposolubles (hydrocarbures naturels (le squalène par exemple), stérols libres (le cholestérol essentiellement), pigments (tels le $\beta$-carotène), vitamines liposolubles (A.D.E.K.).

Certains lipides du lait ne "suivent » donc pas la matière grasse proprement dite lors de l'obtention de celle-ci par des moyens mécaniques. Ces lipides constitués essentiellement de lipides complexes ou lipides polaires sont à la fois lipophiles par la longue chaîne hydrocarbonée des acides gras qu'ils renferment et lipophobes par le groupe phosphoré, aminé, sucré..., qu'ils contiennent également.

Les phospholipides représentent 90 à 92 p. 100 des lipides complexes. Le lait contient 0,3 à $0,4 \mathrm{~g}$ de lipides complexes par litre (1 p. 100 des lipides totaux). Ceux-ci se répartissent en lipides liés à la membrane des globules gras entraînés ultérieurement avec la crème $(2 / 3)$ et en lipides liés à la caséine et maintenus dans le lait écrémé (1/3). Les lipides complexes de la crème " passent " en 
grande partie dans le babeurre au cours de la fabrication du beurre. La faible proportion de phospholipides qui reste dans le beurre $(0,2 \mathrm{~g}$ p. $100 \mathrm{~g}$ de matière grasse) est éliminée presque totalement avec la phase aqueuse, lors de la préparation du « butter-oil » à partir du beurre.

La biosynthèse des lipides complexes, leurs fonctions dans la membrane des globules gras du lait et leur rôle dans la technologie des produits laitiers constituent les aspects sans doute les plus intéressants de l'étude des lipides complexes du lait.

\section{I. - NATURE ET COMPOSITION DES LIPIDES COMPLEXES DU LAIT}

\section{1) Définition et classification}

Les lipides complexes constituent un groupe très diversifié. Ils comprennent un groupe polaire associé à un acide gras. Ce groupe polaire est de nature variée : acide aminé, acide phosphorique, sucre, sphingosine.

On peut diviser les lipides complexes en deux classes :

- les lipides complexes contenant du glycérol ; analogues.

- les lipides complexes contenant de la sphingosine ou des bases

Chacune de ces classes peut à son tour être subdivisée :

- en lipides complexes avec phosphore ;

- en lipides complexes sans phosphore.

Les lipides complexes contenant du glycérol et du phosphore, constituent la classe la plus importante ( 1 p. 100 des lipides totaux) et sont appelés les glycéro-phospholipides (75 p. 100 des lipides complexes).

Appartiennent à ce groupe : la phosphatidyl éthanolamine, la phosphatidyl choline, la phosphatidyl sérine, le phosphatidyl inositol.

Leur formule générale est:

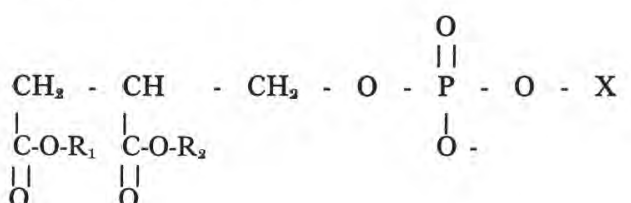

$$
\begin{aligned}
& \mathrm{R} \text { = chaîne carbonée d'acide gras } \quad \mathrm{X} \text { = groupe polaire }
\end{aligned}
$$

Ces composants peuvent exister sous trois formes :

- diacyl : estérification du glycérol par deux acides gras (la plus importante) ; 
- alkoxy-éther : une fonction éther remplace une fonction ester ; liaison.

- vinyl éther : une fonction éther se place en $\alpha$ d'une double

Ces derniers composés sont des plasmalogènes.

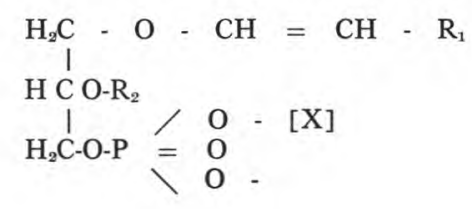

Dans le lait, les lipides complexes contenant du glycérol mais sans phosphore tels que les galactosyl-glycérides, abondants dans les plantes, ou les cardiolipides, qui existent dans le tissu mammaire, ne se rencontrent pas.

La deuxième classe des lipides complexes est constituée par des composés contenant une base (amino-alcool) et principalement la sphingosine $\left(\mathrm{C}_{18} \mathrm{H}_{37} \mathrm{NO}_{2}\right)$ de formule :

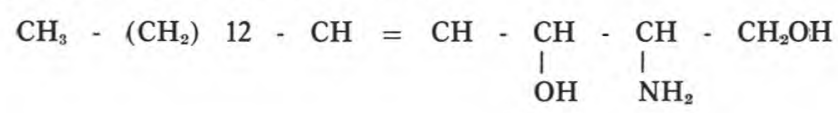

La fonction amine de la sphingosine réagit avec la fonction acide des acides gras pour donner une fonction amide.

Le principal sphingolipide du lait contenant du phosphore est la sphingomyéline ( 20 p. 100 des lipides complexes) de formule générale :

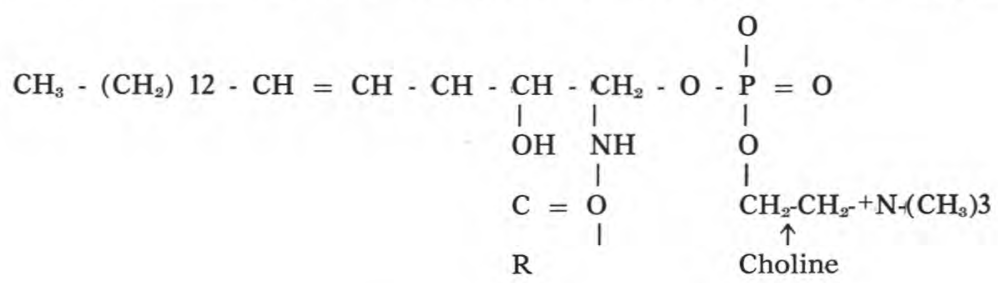

Ce composé peut être aussi appelé céramide phosphoryl-choline, le céramide étant constitué par une molécule de sphingosine liée à un acide gras.

Dans le second groupe (sphingolipides sans phosphore), se trouvent les céramides mono et dihexoses.

Ces composés se rapprochent des cérébrosides (ils possèdent la même formule) mais, à la différence de ces derniers, ils ne renferment 
que de petites quantités ( 1 p. 100 des acides gras totaux des céramides) d'acides hydroxylés.

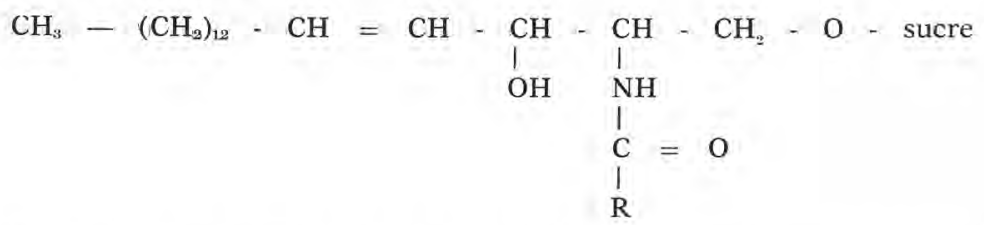

Le lait contient aussi des gangliosides qui ressemblent aux cérébrosides mais qui contiennent plusieurs molécules de sucres et aussi de l'acide neuraminique (ou acide sialique).

\section{2) Composition en acides gras des lipides complexes}

L'absence d'acides gras à courte chaîne, la présence d'acides gras à longue chaîne, une proportion relativement forte d'acide linoléique $\left(\mathrm{C}_{18}: 2\right)$ caractérisent les acides gras des lipides complexes du lait (Cf. tab. 1). La présence d'acides saturés à 22, 23 et 24 atomes de carbone constituent une particularité des phospholipides du lait. On trouve cependant peu d'acide arachidonique, $\mathrm{C}_{20}: 4$, alors que cet acide est abondant dans les phospholipides des tissus animaux.

On remarque une distribution préférentielle de certains acides gras sur la molécule de glycérol. Ainsi tout l'acide arachidonique $\mathrm{C}_{2}: 4$ et 90 p. 100 de l'acide linoléique sont localisés en position 2 ou $\beta$ du glycérol-3-phosphate.

En général l'acide oléique est abondant dans les phospholipides. On le trouve en position 1 ou 2 du glycérophosphate. Les acides saturés tels que l'acide stéarique $\mathrm{C}_{18}: 0$ et l'acide palmitique $\mathrm{C}_{16}: 0$ sont localisés en position 1. Ainsi les acides gras polyinsaturés sont en position 2 et les acides gras saturés en position 1.

La phosphatidyl éthanolamine est le phospholipide le plus insaturé, la phosphatidyl choline, la phosphatidyl sérine et le phosphotidyl inositol sont plus saturés.

La sphingomyéline contient une forte proportion $(80 \mathrm{p} .100$ à 96 p. 100) d'acides gras saturés à longues chaînes : acide palmitique et acides saturés à 22,23 et 24 atomes de carbone. Les acides gras monoinsaturés de la sphingomyéline sont riches en isomères trans. La position de la double liaison est préférentielle en 9-10 pour les cis-monoènes, et peu préférentielle pour les isomères trans.

Morrison et Hay [1] ont identifié 31 bases à longues chaînes parmi les sphingolipides du lait : bases dihydroxy, linéaires ou ramifiées, saturées et insaturées. Parmi les bases dihydroxy insa- 


\section{TABLEAU 1}

Proportions relatives des acides gras des phospholipides totaux et des fractions phospholipidiques du lait de vache

(Résultats exprimés en pourcentage molaire)

Selon Badings [38]

\begin{tabular}{|c|c|c|c|c|}
\hline Acides gras & $\begin{array}{l}\text { Phospholipides } \\
\text { totaux }\end{array}$ & Céphaline & Lécithine & Sphingomyéline \\
\hline $12: 0$ & 0.5 & 0.4 & 0.5 & 0.9 \\
\hline $12: 1$ & $\operatorname{tr}$ & tr & tr & tr \\
\hline 13:0 & $\operatorname{tr}$ & $\operatorname{tr}$ & tr & $\operatorname{tr}$ \\
\hline i $14: 0$ & $\operatorname{tr}$ & $\mathrm{tr}$ & $\mathrm{tr}$ & $\operatorname{tr}$ \\
\hline $14: 0$ & 3,7 & 1,5 & 6,0 & 9,5 \\
\hline $14: 1$ & 0,2 & 0,1 & 0,4 & 0,5 \\
\hline i $15: 0$ & 0,2 & 0,2 & 0,4 & 0,5 \\
\hline $15: 0$ & 0,5 & 0.4 & 1,3 & 1,8 \\
\hline i $16: 0$ & 0,2 & 0,1 & 0,5 & 0,4 \\
\hline $16: 0$ & 15,9 & 9,1 & 30,2 & 30,1 \\
\hline $16: 1$ & 1,2 & 1,2 & 2,1 & 0,7 \\
\hline i $17: 0$ & 0,4 & 0,4 & 1,0 & 0,4 \\
\hline $17: 0$ & 0,5 & 0,5 & 0,8 & 0,5 \\
\hline $17: 1$ & 0,3 & 0,4 & 0,5 & $\operatorname{tr}$ \\
\hline i $18: 0$ & $\mathrm{tr}$ & $\mathrm{tr}$ & 0,1 & $\operatorname{tr}$ \\
\hline 18:0 & 16,3 & 19,1 & 11,6 & 6,3 \\
\hline $18: 1$ & 40,3 & 48,0 & 35,9 & 9,6 \\
\hline $19: 0$ & 0,3 & 0,1 & 0,4 & 0,3 \\
\hline $18: 2$ & 6,1 & 8,9 & 4,7 & 1,2 \\
\hline$?$ & 0,3 & 0,2 & 0,2 & 0,2 \\
\hline $18: 3$ & 1,5 & 1,8 & 1,1 & 0,2 \\
\hline 20:0 & 1,0 & 0,7 & 0,6 & 0,6 \\
\hline $20: 1$ & 0,3 & 0,3 & 0,1 & $\mathrm{tr}$ \\
\hline$?$ & 1,3 & 1,0 & 0,3 & 0,2 \\
\hline $20: 2$ & 0,4 & 0,6 & 0,1 & tr \\
\hline 21:0 & 0,3 & 0,3 & $\operatorname{tr}$ & 0,5 \\
\hline $20: 3$ & 0,9 & 0,8 & 0,4 & 0,2 \\
\hline $22: 0$ & 1,8 & 0,9 & 0,2 & 8,5 \\
\hline$?$ & 0,5 & 0,4 & 0,2 & 0,2 \\
\hline 23:0 & 1,7 & 0,5 & - & 13,1 \\
\hline ? & 0,5 & 0,2 & - & 2,9 \\
\hline ? & 0,1 & $\operatorname{tr}$ & - & 0,3 \\
\hline $24: 0$ & 1,1 & 0,4 & - & 8,2 \\
\hline$\stackrel{?}{2} \cdot 1$ & 1,0 & 0,6 & - & 2,2 \\
\hline ? & $\operatorname{tr}$ & $\operatorname{tr}$ & - & $\operatorname{tr}$ \\
\hline & & & & \\
\hline
\end{tabular}

turées, la sphingénine, ou sphingosine proprement dite comptant 18 atomes de carbone, est la plus importante. Les proportions relatives des acides gras dans les céramides sont similaires aux proportions relatives des acides gras dans la sphingomyéline avec 
cependant une plus forte proportion d'acides gras saturés à 22 , 23 et 24 atomes de carbone.

\section{3) Biosynthèse des lipides complexes}

D’une façon générale le 1,2-diglycéride est le précurseur commun utilisé dans la synthèse des lipides complexes et des triglycérides.

La synthèse du 1,2-diglycéride, de la phosphatidylcholine ou de la phosphatidyl éthanolamine et du phosphatidyl inositol sont rappelés en annexe ainsi que l'interconversion de la sérine de l'éthanolamine et de la choline.

La sphingosine provient de la condensation de l'acide palmitique et de la sérine. La sphingomyéline peut être obtenue par deux processus. Selon le premier processus la sphingosine est acylée et se combine au phosphorylcholine. Selon le deuxième procédé, le phosphorylcholine se combine à la sphingosine libre et l'acylation suit.

Une rétention préférentielle de diglycérides riches en hexaènes se manifeste lors de la formation de la phosphatidyl éthanolamine et de diglycérides riches en tétraènes lors de la formation de la phosphatidyl choline.

La synthèse des cérébrosides se fait de deux manières : soit par acylation d'une base à longue chaîne suivie de la galactosylation, soit par galactosylation de la base, suivie de l'acylation. La présence d'acides hydroxylés facilite l'acylation.

\section{II. - ASPECTS BIOLOGIQUES \\ DE L'ETUDE DES LIPIDES COMPLEXES DU LAIT}

\section{1) Taux de lipides complexes}

Dans le lait le taux varie selon les espèces [2] ; le lait de vache contient relativement peu de phospholipides $(0,3-0,4 \mathrm{~g}$ par litre) si on le compare au lait de chèvre ou de brebis $(0,45-0,50 \mathrm{~g}$ par litre) et surtout au lait de femme (0,6-0,7 g par litre).

Il n'existe que des variations très limitées du taux de phospholipides au sein d'une même espèce animale, en fonction de la race ou du stade de lactation, ou encore de l'alimentation. En général, la saison de pâturage correspond à un taux minimal de phospholipides alors que la période d'alimentation hivernale correspond à un accroissement du taux de phospholipides dans le lait [3].

\section{2) Organisation des lipides complexes et étude de la «membrane» des globules gras}

Dans le lait fraîchement sécrété la plus grande partie (70 p. 100) des lipides complexes se trouve répartie autour du globule gras 
proprement dit. Les phospholipides sont généralement associés à des protéines formant un ensemble lipide-protéines, plus ou moins justement appelé " membrane ". L'épaisseur de cette membrane est de l'ordre de quelques $\mathrm{nm}$ soit de l'ordre du $1 / 1000^{\circ}$ du diamètre des globules gras. Le matériel membranaire représente 1,2 à $1,5 \mathrm{~g}$ par litre de lait [4].

\section{a) Techniques d'Étude DE LA MEMBRANE}

L'isolement de la «membrane » peut être réalisé par barattage d'une crème lavée [5]. Il est également possible de traiter la crème lavée au moyen d'une solution de desoxycholate de sodium [6] ce qui permet de recueillir deux fractions : l'une soluble dans le désoxycholate que l'on peut assimiler à une couche externe de la membrane du globule gras et l'autre insoluble dans le désoxycholate qui correspond à une couche interne.

L'observation directe de la membrane des globules gras du lait est également possible. Ces dernières années, la microscopie électronique a été intensivement utilisée dans ce domaine.

Toutes les techniques d'étude de la membrane du globule gras à l'aide de la microscopie électronique détruisent cependant plus ou moins cette membrane. La technique d'étude par fixation chimique semble se révéler la plus destructive alors que la technique plus récente de cryodécapage respecte davantage la structure primitive [7]. Les opinions varient cependant selon les auteurs.

L'observation peut être effectuée directement sur la crème, ou sur des fragments de membrane, ou encore, si l'on s'intéresse plus précisément au phénomène même de la sécrétion, sur les cellules épithéliales du tissu mammaire.

\section{b) Composition CHIMIQUe DE LA MEMBRANE}

Une revue des résultats analytiques obtenus sur la membrane des globules gras a été présentée par Prentice en 1969 [8]. Les équipes de Brunner et de Thompson $[5,9]$ et de Chien et Richardson [4] et plus récemment de Bracco et Hidalgo [10] se sont intéressées à l'analyse des lipides qui constituent $60 \mathrm{p} .100$ environ du matériel membranaire et comprend des lipides neutres et des phospholipides.

On a présenté dans le tableau 2 la composition de la fraction lipidique de la membrane du lait de vache selon Thompson et al. [5] et selon Bracco et al. [10].

Les lipides neutres les plus polaires (glycérides partiels) les phospholipides, les glycolipides, le cholestérol se concentrent dans la membrane des globules gras.

L'analyse des acides gras révèle une proportion relativement élevée d'acide palmitique et d'acide stéarique. Ceci correspond à 
TABLEAU 2

Composition des lipides de la « membrane » des globules gras du lait (Résultats exprimés en g p. $100 \mathrm{~g}$ de lipides de membrane)

\begin{tabular}{|c|c|c|c|}
\hline \multicolumn{2}{|c|}{$\begin{array}{c}\text { Selon THOMPson et al. } \\
\text { (1961) [5] }\end{array}$} & \multicolumn{2}{|c|}{$\begin{array}{l}\text { Selon BRACCO et al. } \\
\text { (1972) [10] }\end{array}$} \\
\hline $\begin{array}{l}\text { Caroténoïdes } \\
\text { Squalène } \\
\text { Esters de stérol } \\
\text { Triglycérides } \\
\text { Acides gras libres et } \\
\text { autre triglycérides } \\
\text { Cholestérol } \\
\text { Diglycérides } \\
\text { Monoglycérides } \\
\text { Phospholipides }\end{array}$ & $\begin{array}{r}0,45 \\
0,61 \\
0,79 \\
53,41 \\
\\
6,30 \\
5,17 \\
8,14 \\
4,66 \\
20,35\end{array}$ & $\begin{array}{l}\text { Triglycérides } \\
\text { Diglycérides } \\
\text { Monoglycérides } \\
\text { Acides gras libres } \\
\text { Insaponifiable } \\
\text { Phosphore organique }\end{array}$ & $\begin{array}{r}61,70 \\
8,90 \\
\text { traces } \\
6,70 \\
0,89 \\
0,85\end{array}$ \\
\hline
\end{tabular}

la présence de triglycérides à haut point de fusion préférentiellement disposés à la partie externe du globule, c'est-à-dire adjacents à la zone interne de la membrane.

La membrane contient en outre des protéines spécifiques [12] et une grande quantité d'enzymes variées. Dowben et al. [13] ont récemment présenté une étude des enzymes de la membrane des globules gras du lait de vache. Parmi ces enzymes se rencontrent des estérases, des ATPases, la glucose 6-phosphatase, la xanthine oxydase, l'aldolase mais il n'y a pas de lipase dans la membrane du lait fraîchement sécrété.

La présence d'acide ribonucléique dans la membrane des globules gras a été signalée [14]. Mais la présence de ribonucléase dans le lait rend difficile une exacte détermination.

Des métaux, tels le fer et le cuivre sont également présents. Quelques composés isolés, par exemple l'acide thioctique (6-8, dithio octanoïque) ont été signalés dans la membrane des globules gras [15].

Les études de structure se rapportant à l'organisation même des éléments de la membrane sont également intéressantes.

Chien et Richardson [4] ont utilisé l'ultracentrifugation et ont obtenu un fractionnement de la membrane en cinq lipoprotéines d'apparences variées et caractérisées par une composition globale spécifique (proportions relatives de protéines, de lipides neutres et de phospholipides) et un schéma de répartition des acides gras également spécifique. Une proportion élevée de phospholipides 
s'accompagne de la présence d'acides saturés à 22,23 et 24 atomes de carbone caractéristiques des acides gras des phospholipides du lait.

Ainsi, selon un schéma encore classiquement admis $[8,10]$, la membrane des globules gras serait constituée par l'association de deux couches de lipoprotéines de composition et de propriétés différentes : une couche externe riche en enzymes et facilement détachable et une couche interne fortement liée au globule gras proprement dit.

Anderson et al. [17] ont cependant récemment rejeté cette conception en constatant une égale accessibilité de toutes les fractions de membrane à l'effet d'un traitement en surface (marquage radioactif de protéines par exemple).

En outre les études entreprises ces dernières années en vue de déterminer l'origine de la membrane des globules gras, et donc des lipides complexes qu'elle renferme, ont totalement renouvelé les conceptions concernant la structure de la membrane des globules gras.

\section{c) ORIGINE DE LA MEMbRANE DES GLOBULES GRAS}

La mise en évidence de l'origine cellulaire de la membrane des globules gras résulte à la fois des analyses chimiques effectuées sur un matériel cellulaire bien défini et des observations effectuées par microscopie électronique.

\section{a) Résultats des analyses biochimiques}

Une analyse comparée des phospholipides du lait et du tissu mammaire effectuée en 1967 par Parsons et Patton [18] a mis en évidence une similitude de nature des lipides complexes mais non un schéma de répartition équivalent.

Les lipides complexes du lait contiennent en effet plus de phosphatidyléthanolamine, plus de sphingomyéline, plus de céramides, mais moins de phosphatidyl choline, de phosphatidyl sérine, de phosphatidyl inositol que le tissu mammaire et ne contiennent pas de cardiolipides, composés caractéristiques des lipides complexes des membranes mitochondriales (cf. tab. 3).

L'étude comparée des lipides complexes de la membrane des globules gras et des différents organites des cellules sécrétrices a permis d'élucider le problème de l'origine des lipides complexes du lait.

Les membranes des microsomes [19] pauvres en sphingomyéline, plus pauvres en phosphatidyl éthanolamine que les lipides complexes du lait et plus riches en phosphatidyl choline ne peuvent être les précurseurs directs des lipides complexes du lait, comme cela avait été suggéré il y a quelques années par Morton [20]. 


\section{TABLEAU 3}

Composition moyenne des lipides polaires dans le lait de vache

\begin{tabular}{l|c}
\multicolumn{1}{c|}{ Nature des composés } & $\begin{array}{c}\text { Proportions exprimées } \\
\text { en p. 100 du total }\end{array}$ \\
\hline & \\
Diacyl phosphatidyl éthanolamine & 30 \\
Diacyl phosphatidyl choline & 28 \\
Diacyl phosphatidyl sérine & 8 \\
Diacyl phosphatidyl inositol & 5 \\
Vinyl éther phosphatidyl éthanol amine & 1 \\
Vinyl éther phosphatidyl choline & 3 \\
Sphingomyéline & 19 \\
Céramide monohexoside & 3 \\
Céramide dihexoside & 3 \\
& \\
\hline
\end{tabular}

De même les membranes mitochondriales [21] du tissu mammaire, riches en phosphatidyl choline et phosphatidyl éthanolamine mais renfermant en outre une portion élevée $(20$ p. 100) de cardiolipides absents des lipides complexes du lait ne sont pas non plus des précurseurs directs des lipides polaires du lait.

Par contre l'analyse des lipides complexes de la membrane cytoplasmique apporte des éléments pasitifs $[22,23,24]$. En effet la sphingomyéline constitue 33 p. 100 et les céramides 20 p. 100 des lipides complexes totaux.

La contribution des lipides complexes de la membrane cytoplasmique de la cellule sécrétrice est donc essentielle dans la formation des lipides complexes du lait.

Le corps de Golgi, autre élément structural de la cellule, actif dans la synthèse des protéines, semble également jouer un rôle. On a par exemple trouvé dans le lait écrémé de l'U.D.P. galactosyltransférase, enzyme spécifique des corps de Golgi assimilable donc à un "marker» [25].

Les membranes des vésicules de Golgi également riches en cérébrosides pourraient ainsi être les précurseurs des lipides complexes du lait écrémé $[25,26]$.

Les enzymes de la membrane des globules gras sont semblables aux enzymes de la membrane cytoplasmique [27]. Ils ne renferment pas par exemple, les enzymes spécifiques des membranes mitochondriales [22] (cytochrome C réductase et cytochrome C oxydase).

Par ailleurs des études immunologiques [13] ont montré qu'un antisérum préparé à partir du matériel de membrane du globule 
gras s'agglutine avec les érythrocytes, mettant en évidence une composition voisine des deux membranes.

B) Résultats des observations au microscope électronique

Les études récentes histochimiques entreprises à l'aide de la microscopie électronique, ont permis également de renouveler la théorie même de la sécrétion des lipides complexes du lait.

Selon les premières observations faites à l'aide du microscope électronique par Bargmann et Knoop [28] dans le domaine de la

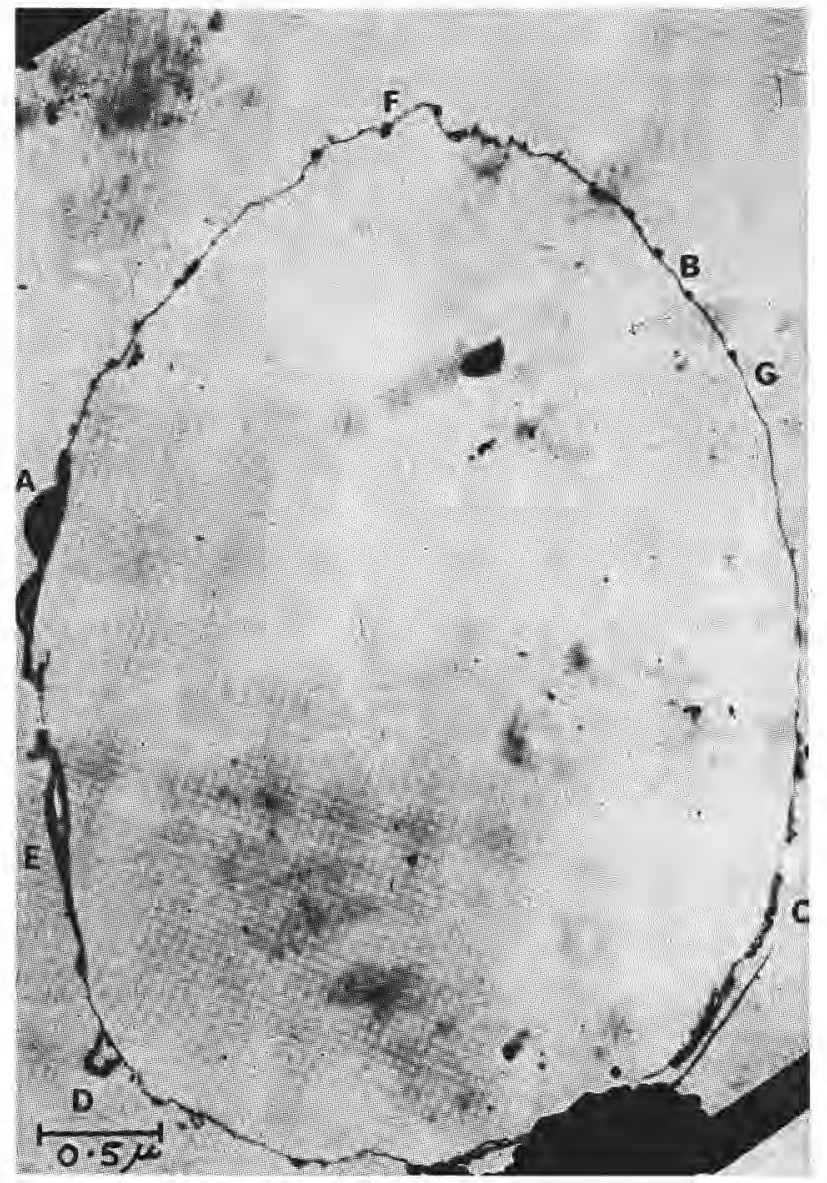

fig. 1

Coupe fine de la membrane d'un globule gras, observée au microscope électronique en A : inclusions cytoplasmiques. Selon Henson et al. [30]. 
sécrétion du lait, le globule gras formé dans la cellule de l'épithélium mammaire, où il provoque une tugerscence est excrété dans la lumière alvéolaire entouré d'une membrane de nature cytoplasmique (unit membrane).

Or l'observation d'une telle membrane, caractérisée sous le microscope électronique par une image trilaminaire (deux zones foncées, une zone claire) s'est révélée très rare lors des observations ultérieures effectuées pourtant dans des conditions améliorées. Selon Knoop [29] il y avait réarrangement de la membrane originelle (dissociation suivie de réadsorption). Toutefois des observations très récentes (1971-1972) faites après utilisation de nouvelles techniques de préparation par exemple au moyen du processus de " cryodézapage " montrent que la présence d'une telle membrane est plus fréquente que cela avait été antérieurement supposé.

Bien que dans le cas le plus général les globules gras apparaissent entourés d'une simple membrane sans structure particulière, Henson et al. [30] ont observé une membrane à trois feuillets bordant la partie externe d'inclusions de nature cytoplasmique adjacente au globule gras proprement dit.

Henson et al. [30] insistent sur l'environnement particulier des inclusions : de chaque côté de la membrane le milieu est aqueux, comme pour toute membrane cellulaire, la structure originale peut être ainsi plus aisément sauvegardée (fig. 1 et 2 ).

Une observation tout aussi capitale que celle de Henson et al. [30] sur les inclusions et assez similaire a été faite par Bauer [31]. En

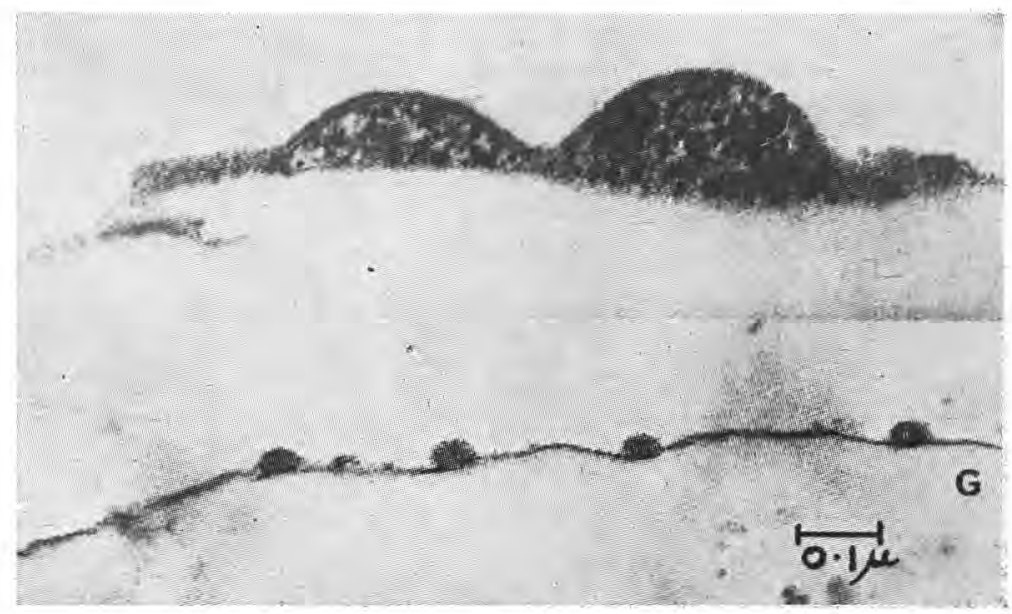

fig. 2

Agrandissement de la zone A représentée sur la figure 1. Une membrane trilaminaire est visible à la partie supérieure des inclusions. Selon HENSON et al. [30]. 
1972 cet auteur révèle la présence de micelles de caséine incluses entre le globule gras et la membrane trilaminaire (fig. 3).

Il existerait ainsi deux membranes de nature différente : l'une entourant le globule gras proprement dit (constituée par un ensemble glycérides à haut point de fusion et phospholipides), l'autre de

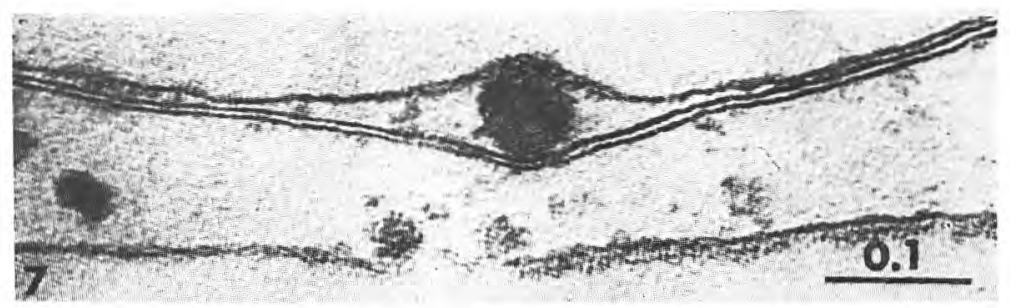

fig. 3

Micelle de caséine incluse entre la matière grasse et la membrane trilaminaire (Cf. BAUER [31])

type trilaminaire bordant l'extérieur d'inclusions de matériel cytoplasmique ou d'inclusions de granules de caséine (fig. 4). Selon Henson et al. [30] les inclusions dériveraient du cytoplasme de la cellule sécrétrice de la glande mammaire et seraient des sites d'activité enzymatique (cf fig. 5).

Les observations de Wooding [32] montrent de manière plus nette encore l'existence dans certains globules d'un matériel cyto-

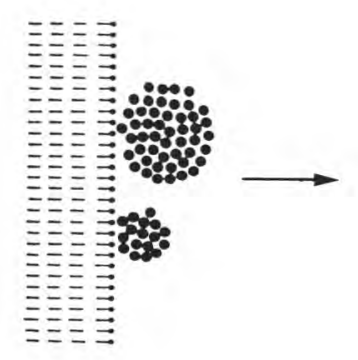

A

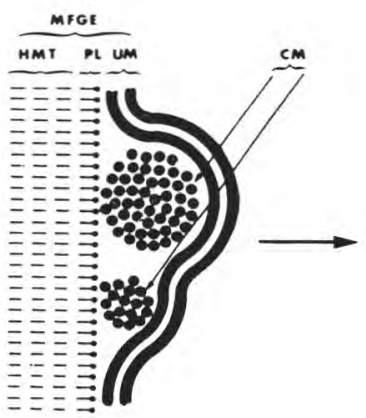

B

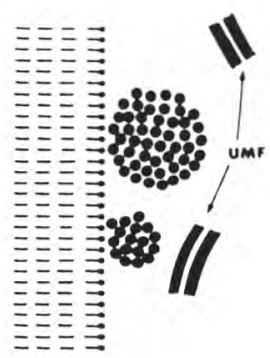

C

fig. 4

Représentation schématique de l'enveloppe des globules gras. Selon BAUER [31]

MFGE : Milk fat globule enveloppe.

$\mathrm{CM}$ : Casein micelles.

HMT : High melting triglycerides.
PL : Phospholipides.

UM : Unit membrane.

UMF : Unit membrane fragment. 
plasmique où s'identifient réticulum endoplasmique, granules de caséine, mitochondries mêmes. Si, selon Wooding [32], pour une raison ou une autre la séparation du globule gras d'avec la cellule sécrétrice se produit avant que la membrane cytoplasmique ait totalement entouré le globule, une fraction de cytoplasme cellulaire est intégrée dans le globule et entraîné avec lui. Des fragments de cytoplasme peuvent ultérieurement migrer dans la phase aqueuse. Certaines observations permettent de supposer que des éléments des corps de Golgi seraient intégrés dans ces fragments (Wooding, 1970).

Selon Patton [33] cependant il n'y aurait pas de sécrétion apocrine ; ces fragments ou débris cytoplasmiques (microvilli et fragments de membrane) observés dans le lait écrémé, qui peuvent en effet contenir des membranes de corps de Golgi, accompagnent selon lui, la sécrétion protéinique (fig. 5).

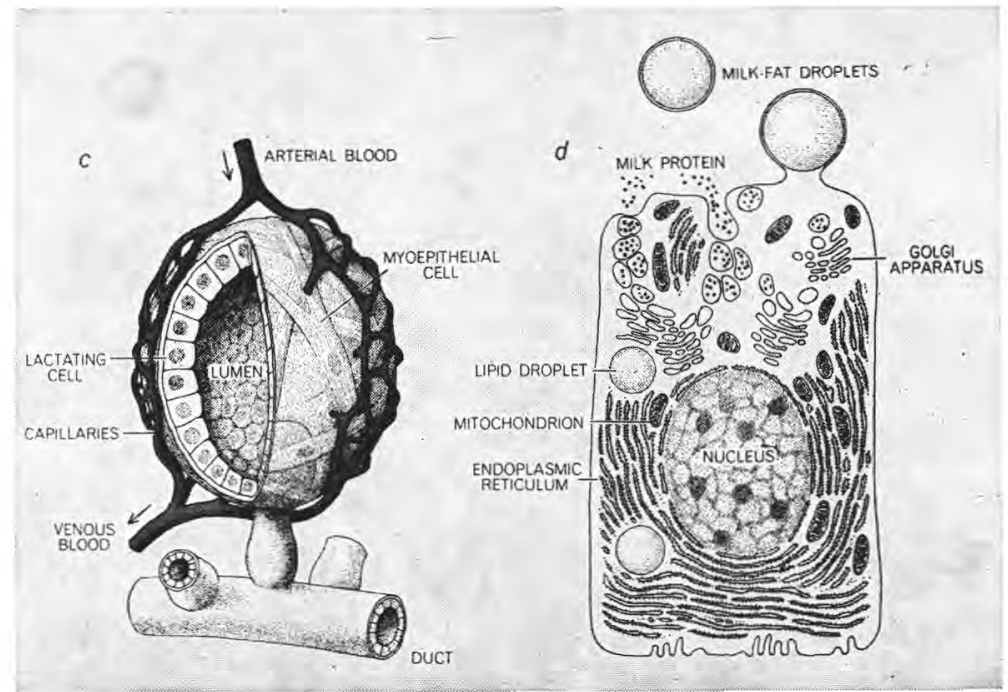

fig. 5

Représentation schématique d'une alvéole avec les cellules secrétrices entourant la lumière (c) et structure détaillée d'une cellule secrétrice (d). Selon Patton [33].

Quoiqu'il en soit l'étude tant structurale qu'enzymatique et biochimique des fragments cytoplasmiques présents dans le lait écrémé apportera sans doute des informations exploitables sur le plan pratique. 


\section{III. - ASPECTS TECHNOLOGIQUES DE L'ETUDE DES LIPIDES COMPLEXES DU LAIT}

\section{1) Nature de l'émulsion. Rôle des lipides complexes}

Dans le lait de vache la matière grasse se présente sous forme de globules de 1 à $10 \mu \mathrm{m}$ de diamètre finement dispersés dans le sérum du lait. Ces globules essentiellement constitués de triglycérides insolubles dans l'eau, forment une émulsion stable. La stabilité est assurée par la présence d'une barrière énergétique à la surface des globules gras qui empêche l'agrégation des globules entre eux. Cette barrière est à la fois mécanique (existence d'une " membrane ") et électrique (le complexe lipoprotéinique possède une charge). La composition chimique et la structure physique de la membrane en surface déterminent sa compacité et sa résistance, facteurs décisifs quant au maintien de l'émulsion lors des différents traitements technologiques.

Ainsi lors de la fabrication du beurre la plus grande partie des lipides complexes se rassemblent dans la crème, puis dans le babeurre et le sérum de beurre.

Si l'on utilise le processus inverse, telle l'addition d'huile de beurre dans du lait écrémé il est difficile d'obtenir une bonne dispersion de la matière grasse dans le lait si l'on n'ajoute pas des agents émulsifiants. La stabilité de l'émulsion dans le lait est due à la présence d'émulsifiants naturels : les lipides complexes.

\section{2) Influence du stade de lactation sur la stabilité de l'émulsion}

$\mathrm{Au}$ fur et à mesure que progresse la lactation, le diamètre moyen des globules gras s'abaisse, leur nombre s'accroît, ce qui requiert un matériel membranaire plus important. Or on a constaté [34] que les taux de phosphatidyl choline et de sphingomyéline (composés majeurs de la membrane des globules gras) diminuent dans le lait au cours des 2 derniers mois de la lactation.

Sans doute, le problème de la rancidité par hydrolyse en fin de lactation est lié à une déficience en matériel de membrane.

\section{3) Effets des traitements technologiques}

a) REFRoIdissEMENT dU LAIT

Le refroidissement du lait (à $4^{\circ} \mathrm{C}$ le plus souvent) est une pratique universellement utilisée.

Les modifications surviennent alors au niveau de la membrane des globules gras : pertes de protéines et de phospholipides originels, adsorption d'une lipase active et de protéines plasmatiques [34]. 
Les modifications survenant dans la composition des protéines de la membrane ont été étudiées en détail récemment par Anderson et al. [35].

Le refroidissement affecte la structure de la membrane du globule gras de telle façon que celle-ci devient plus susceptible aux dommages mécaniques. Les diverses actions qui accompagnent le refroidissement (agitation, variations de température) accentuent le dommage causé et permettent à l'activité lipasique de se manifester.

\section{b) L'HOMOGÉNÉISATION}

L'homogénéisation en réduisant le diamètre moyen des globules gras accroît considérablement leur surface totale. La quantité de matériel membranaire n'étant pas modifiée l'action protectrice qu'exerce ce matériel vis-à-vis du globule gras se trouve affaiblie. Un lait cru homogénéisé rancit rapidement si une pasteurisation n'intervient pas aussitôt après le traitement. Le traitement thermique de pasteurisation doit être suffisamment sévère pour assurer la destruction de la lipase naturelle du lait c'est-à-dire par exemple $15 \mathrm{~s}$ à $74^{\circ} \mathrm{C}$ [36] alors que $15 \mathrm{~s}$ à $72^{\circ} \mathrm{C}$ constituent les conditions thermiques suffisantes pour obtenir la destruction de la phosphatase.

\section{c) LA CONCENTRATION DU LAIT}

La concentration du lait est un processus technologique qui précède parfois la fabrication de fromages (procédé Stenne-Hutin par exemple). On observe alors même si le lait concentré est ramené à son volume initial par dilution, une rétention de matière grasse dans le caillé. Selon Cheeseman et Mabitt [37] ceci est dû non pas au processus de concentration per se mais à la formation d'un complexe entre les micelles de caséine (caséine $\mathrm{K}$ ) et la membrane du globule gras. Cette combinaison est facilitée ou même provoquée par le refroidissement et l'agitation antérieures du lait.

\section{4) Conséquences de l'organisation des lipides complexes}

\section{a) FABRICATION DU BEURRE}

La fraction protéinique du beurre est constituée par une forte proportion de matériel membranaire. Le taux de phospholipides dans le beurre de baratte est de 0,2 p. 100 environ des lipides totaux (soit 15 p. 100 du matériel non gras sec) mais dans un beurre Golden Flow ce taux peut s'abaisser à 0,05 p. 100 des lipides totaux.

La structure du beurre est alors différente, et ses propriétés mécaniques (levage de pâte feuilletée par exemple) sont réduites.

Le cuivre est un catalyseur d'oxydation. Le lait contient du cuivre naturel dont une forte proportion est liée aux protéines de la membrane, mais ce cuivre, grâce sans doute à une « protection " 
particulière n'est pas en cause dans le phénomène d'oxydation. Par contre le cuivre de contamination qui provient du matériel utilisé en laiterie s'amasse dans le lait. Lié aux protéines du sérum, il peut être adsorbé sur les protéines de la membrane au cours de l'acidification de la crème. Il se crée ainsi des risques accrus d'oxydation par un rapprochement enzyme-substrat.

$\mathrm{Pa}$ : contre au cours de la pasteurisation de la crème les protéines de membrane subissent une dénaturation et le cuivre adsorbé peut, en partie, être détaché avec une fraction de matériel membranaire. C'est une des raisons pour lesquelles on constate une amélioration de la conservation du beurre avec un traitement thermique plus sévère des crèmes (cf fig. 6) [16].

\section{b) Fabrication DES POUdRES}

Pour être de bonne qualité les poudres grasses, de lait ou de tout autre matériel biologique doivent posséder une bonne mouillabilité et une bonne stabilité de la matière grasse. Les deux problèmes sont liés. En effet la mouillabilité est d'autant meilleure que la surface de la poudre est plus hydrophile et plus la surface est hydrophile plus la matière grasse est protégée et donc stable.

Pour obtenir ces conditions il est nécessaire d'éviter toute rupture de la membrane des globules gras de telle façon que le pouvoir de " cohésion " que possèdent les lipides complexes soit maintenu et que la matière grasse (huile) n'exsude pas au dehors risquant alors par refroidissement de recouvrir les protéines et de créer une surface hydrophobe altérable.

La richesse en lipides complexes du babeurre explique au moins partiellement la facilité avec laquelle ce produit, transformé en poudre, se conserve.

Un taux minimum de lipides extractibles par un solvant apolaire constitue ainsi un "critère " permettant une estimation de la qualité d'une poudre.

\section{5) Les lipides complexes et les altérations}

a) LiPolyse

Ce problème a été évoqué à diverses reprises au cours de l'exposé. Il y a lipolyse s'il y a contact entre l'enzyme (lipase, lipoprotéine lipase) et le substrat.

Les lipides complexes ne jouent pas un rôle direct dans la lipolyse. Toutefois lors du passage des lipides complexes de la membrane cytoplasmique dans le lait des éléments (activateurs de permettre le déclenchement d'une réaction enzymatique.

lipases ? Lipoprotéines particulières ?) peuvent les accompagner et 


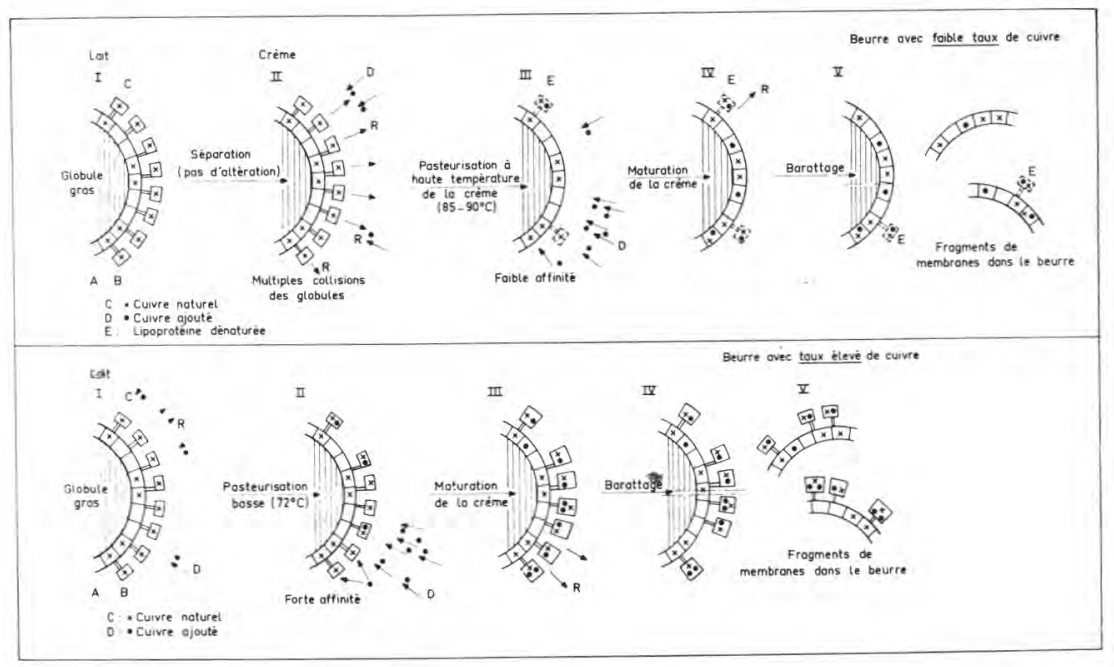

fig. 6

Représentation schématique d'une modification de la structure de la membrane du globule gras au cours de la pasteurisation normale et à haute température. Selon Copius-PeEReboom [16]. (Cf. texte).

A : couche interne de lipoprotéine du globule gras contenant un taux relativement faible de phospholipides.

B : particules de lipoprotéines avec un taux élevé de phospholipides.

C : cuivre naturel.

D : cuivre ajouté.

E : perte de particules de lipoprotéines.

$\mathrm{R}$ : particule de lipoprotéines contenant des protéines dénaturées et des enzymes.

\section{Partie supérieure}

Vue schématique de l'altération dans la structure de la membrane du globule gras au cours du traitement à haute température $\left(82-90^{\circ} \mathrm{C}\right)$ d'une crème préparée à partir de lait non pasteurisé et au cours du barattage ultérieur.

A $82-90^{\circ} \mathrm{C}$ les forces de liaison entre les lipoprotéines et la zone interne de la membrane s'atténuent et des particules lipoprotéiniques quittent la membrane en entraînant du cuivre.

\section{Partie inférieure}

Vue schématique de l'altération dans la structure de la membrane du globule gras au cours de la pasteurisation à température normale $\left(72^{\circ} \mathrm{C}\right)$ et au cours du barattage ultérieur.

$\mathrm{Au}$ cours de la pasteurisation, le cuivre de contamination peut être adsorbé sur la membrane (zone extérieure riche en phospholipides) ; mais au cours de la pasteurisation la couche externe de la membrane des globules gras est peu altérée et une très faible quantité de lipoprotéines quittent la membrane. $\mathrm{Au}$ cours de la maturation se produit une migration du cuivre de contamination de la phase aqueuse vers la membrane. Au cours du barattage ultérieur, une faible proportion de particules lipoprotéiriques est éliminée par agitation mécanique, et la presque totalité du cuivre reste associée aux éléments de la surface de la membrane des globules gras qui sont ensuite transférés dans le beurre. 


\section{b) OXYDATION}

Si l'on a souvent affirmé dans les années antérieures que l'oxydation des lipides du lait portait préférentiellement sur les acides polyinsaturés des phospholipides, ce point n'a pas été ultérieurement confirmé. On admet aujourd'hui que plus que la nature des lipides impliqués (lipides complexes ou lipides simples) compte l'arrangement moléculaire qui permet ou ne permet pas le départ de la réaction.

\section{IV. - CONCLUSION}

Les recherches actuelles concernant les lipides complexes sont nombreuses. Les études de composition se développent. Elles concernent surtout les lipides complexes mineurs, les céramides par exemple. Mais les développements des recherches, dans le domaine de l'organisation au niveau moléculaire des lipides complexes du lait et de leurs liaisons avec les autres éléments de la membrane des globules gras, revêtent un grand intérêt. Selon les modalités de la sécrétion des lipides du lait, des modifications pourraient apparaître dans le comportement ultérieur de ces lipides (susceptibilité à la lipolyse, à l'oxydation, etc.) par suite d'une composition différente des constituants de la membrane des globules gras (enzymes, activateurs ou inhibiteurs d'activité enzymatique, etc.).

\section{Rés u m é}

\section{LES LIPIDES COMPLEXES DU LAIT DE VACHE ASPECTS BIOLOGIQUES. ASPECTS TECHNOLOGIQUES}

La nature et la composition des lipides complexes du lait de vaches ont été rappelées.

Les résultats des recherches entreprises ces dernières années par la voie de l'analyse biochimique ou par la voie de la microscopie électronique ont fait rapidement progressé les connaissances concernant les modalités de la sécrétion des lipides du lait au niveau cellulaire.

Certains problèmes technologiques ou certaines modifications physico-chimiques et organoleptiques des lipides du lait sous l'effet de facteurs physiques externes (actions thermiques, mécaniques) ou de facteurs internes liés au phénomène secrétoire sont ensuite plus spécifiquement étudiés, afin de mettre en évidence le rôle joué par certains constituants du lait ou de la membrane des globules gras qui ont pour origine la membrane plasmatique de la cellule secrétrice. 


\section{S u m m a r y \\ THE COMPLEX MILK LIPDS \\ BIOLOGICAL AND TECHNOLOGICAL ASPECTS}

The authors recall the composition of the complex milk lipids and the principle of their biosynthesis. They emphasize the dynamic aspect of these lipids. Some of their constituents are derived from the cytoplasmic membrane of the secretory cells, and at the same time as the "membrane " of the fat globules undergoes rearrangements of all kinds, the complex milk lipids become either isolated or are integrated into the lipoproteins, and finally distributed between the aqueous and lipid phase.

The effect of technological treatments on the organization of complex lipids and on their distribution, and the specific role played by these lipids in the maintenance of the quality or structure of the products are discussed.

\section{Annexes}

I. - FoRMULE GÉr.ÉRALE D'UN PHOSPHOLIPIDE

$$
\begin{aligned}
& \mathrm{CH}_{2}-\mathrm{O}-\mathrm{CO}-\mathrm{R}_{1} \\
& \mathrm{CH}-\mathrm{O}-\mathrm{CO}-\mathrm{R}_{2} \\
& \mathrm{CH}_{2}-\mathrm{O}-\mathrm{P}=\stackrel{\mathrm{OH}}{\mathrm{O}} \text { ar ac phosphatidique }
\end{aligned}
$$

$$
\begin{aligned}
& \mathrm{CH}_{2}-\mathrm{O}-\mathrm{CO}-\mathrm{R}_{1} \\
& \mathrm{CH}-\mathrm{O}-\mathrm{CO}-\mathrm{R}_{2} \\
& \mathrm{CH}_{2}-\mathrm{O}-\mathrm{P} \stackrel{\mathrm{O}}{\mathrm{O}} \mathrm{O} \\
& \mathrm{O}
\end{aligned}
$$

Phosphatidyl choline (lécithine)

$$
\begin{aligned}
& \mathrm{CH}_{2}-\mathrm{O}-\mathrm{CO}-\mathrm{R}_{1} \\
& \mathrm{CH}-\mathrm{O}-\mathrm{CO}-\mathrm{R}_{2}
\end{aligned}
$$

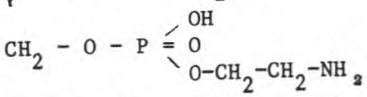

$$
\begin{aligned}
& \mathrm{CH}_{2}-\mathrm{O}-\mathrm{CO}-\mathrm{R}_{1} \\
& \begin{array}{ll}
\mathrm{CH}-\mathrm{O}-\mathrm{CO}-\mathrm{R}_{2} . & \text { Phosphatidyl sérine } \\
\mathrm{CH}_{2}-\mathrm{O}-\mathrm{P} \stackrel{\mathrm{OH}}{\mathrm{O}} \begin{array}{l}
\mathrm{O} \\
\mathrm{O}
\end{array}-\mathrm{CH}_{2}-\mathrm{CH}_{1}-\mathrm{COOH} &
\end{array} \\
& \mathrm{NH}_{2}
\end{aligned}
$$

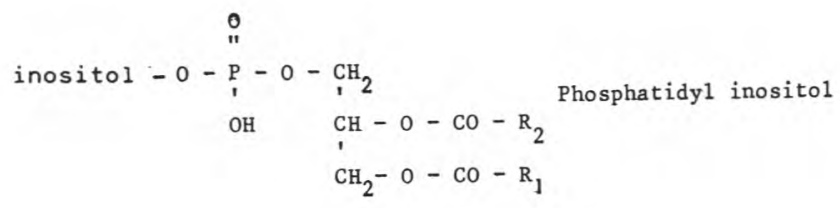


II. - SyNTHÈSE DU 1,2 DIGLYCÉRIDE

\begin{tabular}{|c|c|c|c|c|}
\hline $\mathrm{CH}_{2} \mathrm{OH}$ & & $\mathrm{CH}_{2} \mathrm{OH}$ & & $\mathrm{CH}_{2}-\mathrm{O}-\mathrm{CO}-\mathrm{R}$ \\
\hline & g1ycérol & & $+2 \mathrm{R}-\mathrm{CO}-\mathrm{COA}$ & \\
\hline $\begin{array}{l}\mathrm{CH}-\mathrm{OH} \\
\text {, }\end{array}$ & kinase & $\begin{array}{l}\mathrm{CH}-\mathrm{OH} \\
\text { ? }\end{array}$ & $\overrightarrow{\text { acyl transférase }}$ & $\begin{array}{l}\mathrm{CH}-\mathrm{O}-\mathrm{CO}-\mathrm{R} \\
\text {, }\end{array}$ \\
\hline $\mathrm{I}_{2} \mathrm{OH}$ & & $\mathrm{CH}_{2} \mathrm{O}$ (P) & & $\mathrm{CH}_{2}-\mathrm{O}-(\mathrm{P}$ \\
\hline
\end{tabular}

Ac. glycérophosphorique

Ac. phosphatidique

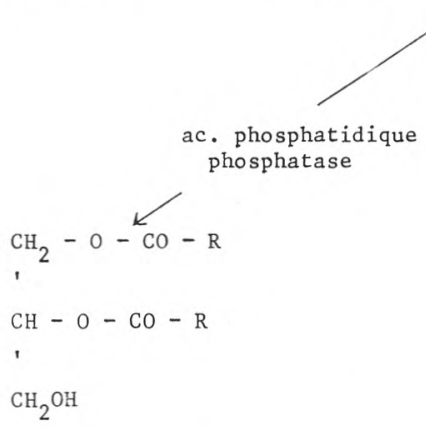

1,2 diglycéride

III. - SyNTHÈSE DE LA PHOSPHATIDYL CHOLINE OU DE LA PHOSPHATIDYL ÉTHANOLAMINE

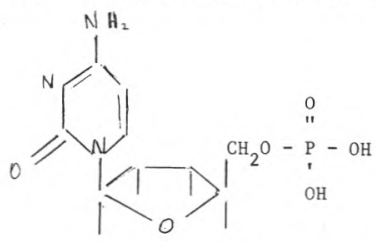

C M P (cytidine monophosphate)
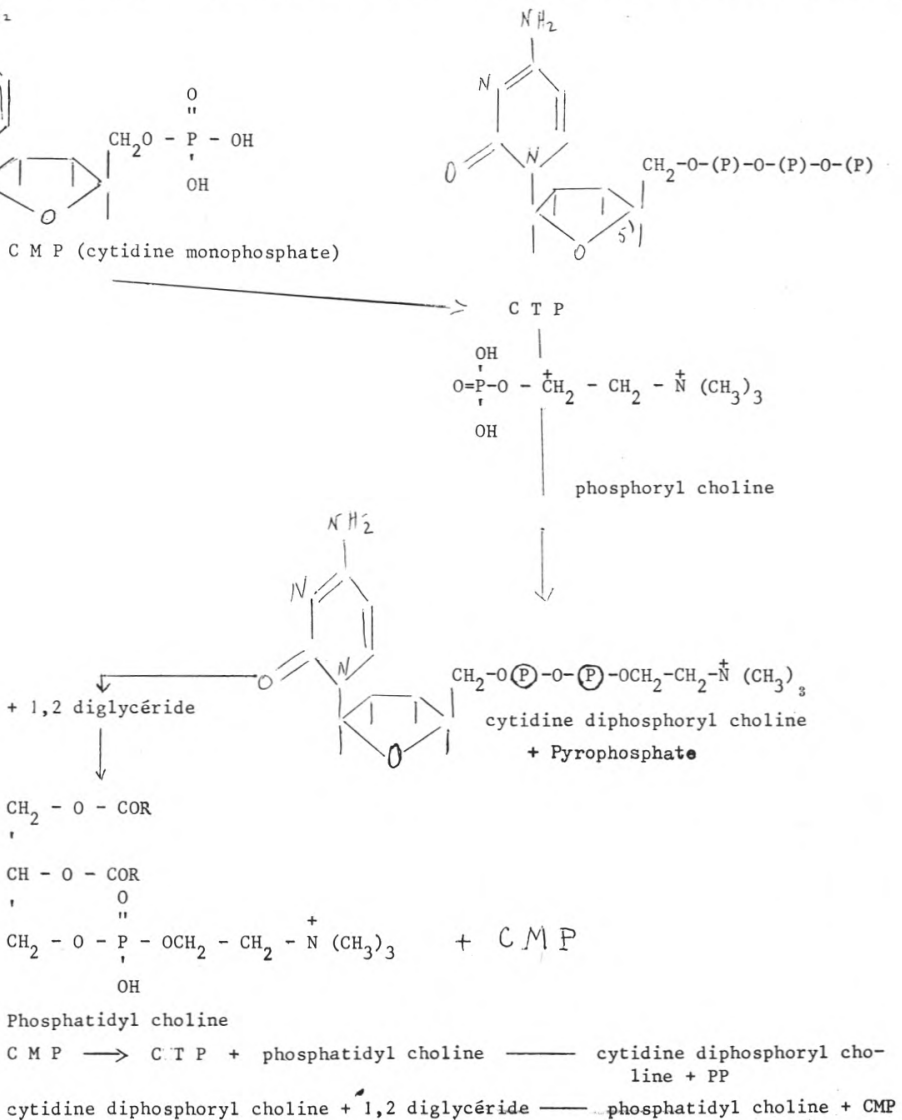


\section{IV. - SYNTHÈSE DU PHOSPHATIDYL INOSITOL}

Acide phosphatidique + C T P — C M P phosphoryl diglyceride + PP

C M P-phosphoryl diglycéride + Inositol — phosphatidyl inositol + CMP

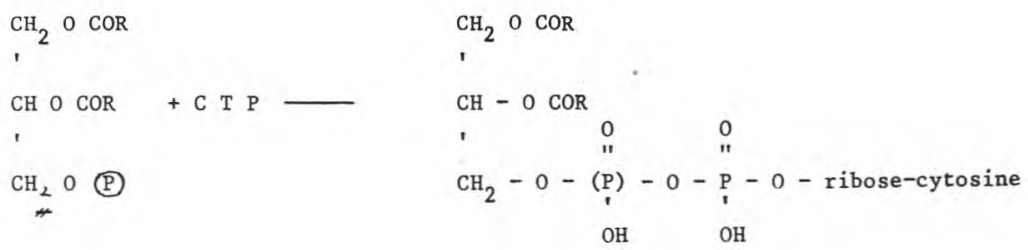

Interconversion de la sérine de l'éthanolamine et de la choline

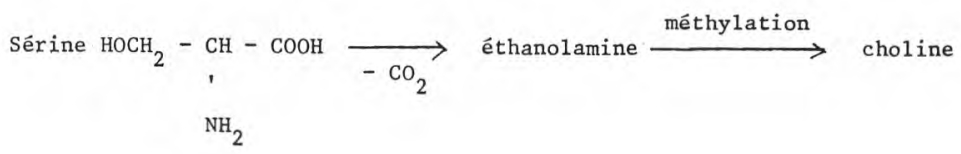

\section{Références bibliographiques}

[1] Morrison (W. R.), Hay (J. D.) (1970). - Biochim. Biophys. Acta, 202, 460.

[2] Kuzdzal-Savoie (S.) (1971). - Ann. Nutr. Alim., 25, A 225.

[3] Rawat (R. S.) (1963). - Agra Univ. J. Res. (ser. Sci.), 12, 245.

[4] Chien (H. C.), Richardson (T.) (1967). - J. Dairy Sci., 50, 451.

[5] Thompson (M. P.), Brunner (J. R.), Stine (C. M.), Lindouist (K.) (1961). J. Dairy Sci., 44, 1589.

[6] Hayashi (S.), Smith (L. M.) (1965). - Biochemistry, 4, 2550.

[7) BaUER (H.) (1972). - J. Dairy Sci., 55, 1375.

[8] Prentice (J. H.) (1969). - Dairy Sci. Abstr., 31, 353.

[9] Brunner (J. R.) (1962). - J. Dairy Sci., 45, 943.

[10] Bracco (U.), Hidalgo (J.), Bohren (H.) (1972). - J. Dairy Sci., 55, 165.

[11] Chien (H. C.), Richardson (T.) (1967). - J. Dairy Sci., 50, 1868.

[12] Herald (C. T.), Brunner (J. R.) (1957). - J. Dairy Sci., 40, 948.

[13] Dowben (R.M.), Brunner (J. R.), Philpott (D. E.) (1967). - Biochim. Biophys. Acta, 135, 1.

[14] Swope (F. C.), Brunner (J. R.) (1965). - J. Dairy Sci., 48, 1705.

[15] Bingham (R. J.), Hiber (J. D.), Aurand (L. W.) (1967). - J. Dairy Sci., 50, 318.

[16] Copius-Peereboom (J. W.) (1969). - Fette, Seifen Anstrich, 71, 314.

[17] Anderson (M.), Cheeseman (G. C.), Knight (D.) (1972). - J. Dairy Res., 39, 409.

[18] Parsons (J. G.), Patton (S.) (1967). - J. Lipid. Res., 8, 696.

[19] Kinsella (J. E.) (1972). - Lipids, 7, 165. 
[20] Morton (R. K.) (1953). - Biochem. J., 57, 231.

[21] Huang (C. M.), Keenan (T. W.) (1971). - J. Dairy Sci., 54, 1395.

[22] Keenan (T. W.), Olson (D. E.), Mollenhauer (H. H.) (1971). - J. Dairy Sci., 54, 295.

[23] Kinsella (J. E.) (1971). - J. Dairy Sci., 54, 1014.

[24] Keenan (T. W.), Morre (D. J.), Olson (D. E.), Yunghans (W. N.), Patton (S.) (1970). - J. Cell. biol., 44, 80.

[25] Patton (S.), Keenan (T. W.) (1971). - Lipids, 6, 58.

[26] Patton (S.), Puppione (D. L.), Stewart (P. S.) (1972). - Z. Zell forsch. mikrosk Anat., 123, 161 ou D.S.A., 34, $\mathrm{n}^{\circ} 2642$.

[27] Patton (S.), Trams (E. G.) (1971). - FEBS Lettres, 14, 230.

[28] Bargmann (W.), Knoop (A.) (1961). - Z. Zellforsch. Mikroskopische Anat., 49, 344.

[29] Knoop (E.), Wortmann (K.) (1959). - Deutsch. Molkerei Z., 80, 491.

[30] Henson (A. F.), Holdsworth (G.), Chandan (R. C.) (1971). - J. Dairy Sci., $54,1752$.

[31] Bauer (H.) (1972). - J. Dairy Sci., 55, 1375.

[32] Wooding (F. B. P.), Peaker (M.), Linzell (J. L.) (1970). - Nature (London), 226, 762.

[33] Patton (S.) (1969). - Scientific American, 221, 59.

[34] Koops (J.), Tarassuk (N. P.) (1959). - Neth Milk Dairy J., 13, 180.

[35] Anderson (M.), Cheeseman (G. C.), Knight (D. J.), Shipe (N. F.) (1972). J. Dairy Res., 39, 95.

[36] Stadhouders (J.) (1961). - Neth. Milk Dairy J., 15, 10.

[37] Cheeseman (G. C.), Mabbitt (L. A.) (1968). - J. Dairy Res., 35, 135.

[38] Badings (H. T.) (1962). - Neth. Milk Dairy J., 16, 217. 